\title{
Publisher Correction: Nanoimaging: Reflected phonons reveal strong coupling
}

Bill Barnes (D)

Correction to: Nature Photonics https://doi.org/10.1038/s41566-021-00773-3, published online 23 February 2021.

In the version of this News \& Views originally published, the name Bylinki used throughout the text and in ref. 2 was incorrect; it should have read Bylinkin. This has now been corrected in the online versions of this News \& Views.

Published online: 12 March 2021

https://doi.org/10.1038/s41566-021-00794-y

(C) The Author(s), under exclusive licence to Springer Nature Limited 2021 